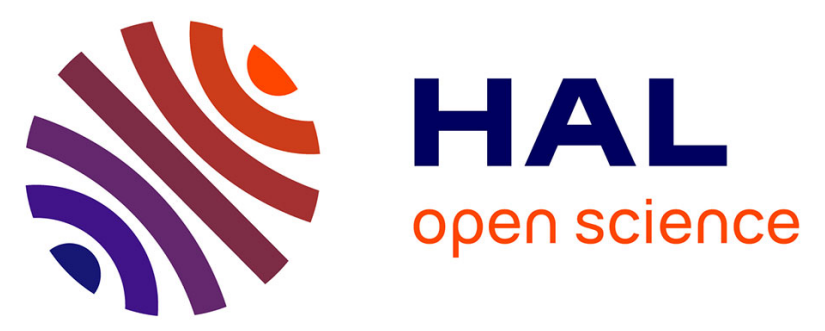

\title{
Breaking the Efficiency Barrier for Ambient Microwave Power Harvesting With Heterojunction Backward Tunnel Diodes
}

\author{
Carlos Henrique Petzl Lorenz, Simon Hemour, Wenjun Li, Yi Xie, Jules \\ Gauthier, Patrick Fay, Ke Wu
}

\section{To cite this version:}

Carlos Henrique Petzl Lorenz, Simon Hemour, Wenjun Li, Yi Xie, Jules Gauthier, et al.. Breaking the Efficiency Barrier for Ambient Microwave Power Harvesting With Heterojunction Backward Tunnel Diodes. IEEE Transactions on Microwave Theory and Techniques, 2015, 63 (12), pp.4544 - 4555. 10.1109/TMTT.2015.2495356 . hal-01722684

\section{HAL Id: hal-01722684 \\ https://hal.science/hal-01722684}

Submitted on 1 Jun 2021

HAL is a multi-disciplinary open access archive for the deposit and dissemination of scientific research documents, whether they are published or not. The documents may come from teaching and research institutions in France or abroad, or from public or private research centers.
L'archive ouverte pluridisciplinaire HAL, est destinée au dépôt et à la diffusion de documents scientifiques de niveau recherche, publiés ou non, émanant des établissements d'enseignement et de recherche français ou étrangers, des laboratoires publics ou privés. 


\title{
Breaking the Efficiency Barrier for Ambient Microwave Power Harvesting With Heterojunction Backward Tunnel Diodes
}

\author{
Carlos Henrique Petzl Lorenz, Student Member, IEEE, Simon Hemour, Member, IEEE, \\ Wenjun Li, Yi Xie, Jules Gauthier, Patrick Fay, Senior Member, IEEE, and Ke Wu, Fellow, IEEE
}

\begin{abstract}
Harvesting low-density ambient microwave power as an alternative power source for small ubiquitous wireless nodes has been proposed in recent papers discussing emerging technologies like the Internet of Things and Smart Cities. However, a literature review of the state-of-the-art Schottky diode based microwave rectifiers shows that a maximum efficiency has been reached for such devices operating in the low-power regime, as is the case for ambient microwave power-harvesters. This work examines the underlying physical mechanisms responsible for this RF-to-dc power conversion efficiency limitation, and explores a high I-V curvature backward tunnel diode to overcome this efficiency limitation. Measurements of the $2.4 \mathrm{GHz}$ RF-to-dc power conversion efficiency at $-40 \mathrm{dBm}$ input power demonstrates that the backward tunnel diode outperforms the HSMS-285B Schottky diode by a factor of 10.5 and the Skyworks SMS7630 by a factor of 5.5 in a lossless matching network scenario. A prototype built using a new GSG probe embedded with a matching circuit showed a total power conversion efficiency of $3.8 \%$ for $-40 \mathrm{dBm}$ input power and $18.2 \%$ for $-30 \mathrm{dBm}$ input power at $2.35 \mathrm{GHz}$.
\end{abstract}

Index Terms-Backward tunnel diode, microwave power harvesting, microwave power rectification, rectenna, Schottky diode.

\section{INTRODUCTION}

T HE way the internet is perceived by its users is going through a significant transformation. Today, nearly two billion people have access to the internet [1], using it to browse websites, play games, work, send and receive e-mails and messages, among many other on-line applications. Most of these applications, however, share a common characteristic: an end-user terminal. Nonetheless an important revolution is in progress, in which ubiquitous objects will be more and more connected to the internet or other local networks, leading to the Internet of Things (IoT) [1], [2]. Some emerging applications of this new

Manuscript received July 01, 2015; revised September 30, 2015; accepted October 13, 2015. Date of publication November 18, 2015; date of current version December 02, 2015. This work was supported in part by the Natural Sciences and Engineering Research Council of Canada (NSERC) and CREATE PERSWADE Training Program. This paper is an expanded version from the IEEE MTT-S International Microwave Symposium, Phoenix, AZ, USA, May 17-22, 2015

C. H. P. Lorenz, S. Hemour, J. Gauthier, and K. Wu are with the Poly-Grames Research Center, École Polytechnique de Montréal, Montreal, QC H3T 1J4, Canada (e-mail: carlos.lorenz@polymtl.ca).

W. Li, Y. Xie, and P. Fay are with the Department of Electrical Engineering, University of Notre Dame, Notre Dame, IN 46556 USA (e-mail: pfay@nd.edu).

Color versions of one or more of the figures in this paper are available online at http://ieeexplore.ieee.org.

Digital Object Identifier 10.1109/TMTT.2015.2495356 paradigm in networking include monitoring the structural health of buildings and monuments, smart roads to help detect heavy traffic so that efficient itineraries can be suggested and traffic light timings can be optimized, remote health monitoring of patients, to cite just a few [3].

Emerging hardware technologies are being studied to enable these Smart Cities, Smart Building Automation and Monitoring, as well as Wearable Devices applications. These usually require wireless connections and small form-factor energy sources to remotely power the ubiquitous sensing and controlling devices, without the burden of a heavy and bulky battery that may be difficult to replace - as in the case of body implants and sensors embedded into building structural parts. Resonant power transfer technology has shown to be a good alternative when a charger may be placed in close proximity to the wireless device of interest, as is the case for parked electric vehicles and even body implants that may be charged at periodic intervals [4]. However, very short operational distances between the charger and device becomes a fundamental obstacle when omnipresent, distributed sensor networks are deployed. Microwave Power Harvesting (MPH) has been proposed as an alternative wireless power source for such low power consumption and low duty cycle ubiquitous devices [5]-[7].

However, an overview of the state of the art in the field of microwave power harvesting suggests that a limitation in the efficiency of Schottky diode-based converters at low incident power levels has been reached [5], [8], [9]. In practice, microwave power rectifiers capable of operating below $-30 \mathrm{dBm}$ are needed for ambient microwave power harvesting (AMPH) as indicated in recent ambient microwave power density assessments [5], [10]. However, to date RF-to-dc conversion efficiency has not yet reached even $10 \%$ at frequencies above 1 $\mathrm{GHz}$ at such low-density power levels. Overcoming this performance barrier is essential for AMPH systems to become a practical reality.

Schottky diodes rely on the thermionic emission, which limits the zero-bias current responsivity $\Re_{I 0}$ to $19.34 \mathrm{~A} / \mathrm{W}$ at 300 $\mathrm{K}$ [8]. As shown in [8], [9], the rectification efficiency of microwave rectifiers operating in the diode's square-law region is directly proportional to the square of $\Re_{I 0}$. Backward tunnel diodes, which make use of quantum mechanical tunneling rather than thermionic emission, have been reported that overcome the Schottky diodes' inherent $\Re_{I 0}$, reaching values near $24 \mathrm{~A} / \mathrm{W}$ for devices optimized for millimeter-wave operation [8], [11] or 
even as high as $35 \mathrm{~A} / \mathrm{W}$ for lower-frequency devices [12]. This paper presents a deeper evaluation of the application of such diodes to overcome the low-power RF-to-dc conversion efficiency limitation observed with Schottky diode microwave rectifiers, an idea which was first introduced in [13] and is extended in this paper. A comparison between a backward diode and an HSMS-285B Schottky diode is presented, demonstrating an increase in RF-to-dc conversion efficiency at extremely low incident power levels, as required when harvesting ambient power below $-30 \mathrm{dBm}$.

\section{Microwave Power Harvesting}

In order to explore possible ways of increasing the power conversion efficiency PCE (1) of ambient microwave power harvesters, it is, first of all, necessary to understand the mechanisms that play a role in the energy rectification process at very low input powers levels, as found in ambient microwave power harvesting scenarios. In such applications, the expected operation power range has maximum peaks reaching $-15 \mathrm{dBm}$ in very high power density areas, with a typical average power of -30 $\mathrm{dBm}$ and below [5], [10]. At such power levels, the square-law microwave power rectification model presented in [9] can be used as a good approximation.

In this rectification model, the power conversion mechanism is sub-divided into different steps, each one with an intrinsic efficiency. These efficiencies combine to produce the total rectifier's PCE, as summarized below:

$\begin{array}{ll}\eta_{m} & \text { is the matching network efficiency; } \\ \eta_{p} & \text { is the parasitic efficiency; } \\ \eta_{0} & \text { is the non-linear junction RF-to-dc } \\ & \text { power conversion efficiency; } \\ \eta_{d c T} & \text { is the dc power transfer efficiency, } \\ & \begin{array}{l}\text { which gives the percentage of the } \\ \text { total rectified dc power that is actually } \\ \text { delivered to the load. }\end{array}\end{array}$

The total power conversion efficiency PCE can thus be calculated using (1):

$$
P C E=\frac{P_{L O A D}}{P_{R F}}=\eta_{m} \cdot \eta_{p} \cdot \eta_{0} \cdot \eta_{d c T}
$$

where $P_{L O A D}$ is the dc power delivered to the load and $P_{R F}$ is the input RF power. The non-linear device characteristics influence in different ways each of these efficiencies. In the case of diodes, which are the subject of the study presented in this work, the Shockley diode model with package parasitic components is used, as shown in Fig. 1. In this figure, $R_{j}$ represents the diode's non-linear junction resistance, $C_{j}$ the junction capacitance, $C_{p}$ the package parasitic capacitance, $L_{p}$, the package inductance, and $R_{S}$ the series resistance.

Using this model, the total $\eta_{0} \cdot \eta_{d c T}$ efficiency when the load connected to the diode is equal to $R_{j 0}$ is given by [9]:

$$
\eta_{0} \cdot \eta_{d c T}=\left(P_{j} \frac{\Re_{I 0}^{2} \cdot R_{j 0}}{4}\right)
$$

where $P_{j}$ is the microwave power delivered to the junction resistance, $R_{j 0}$ is the junction resistance at zero-bias, and $\Re_{I 0}$ is

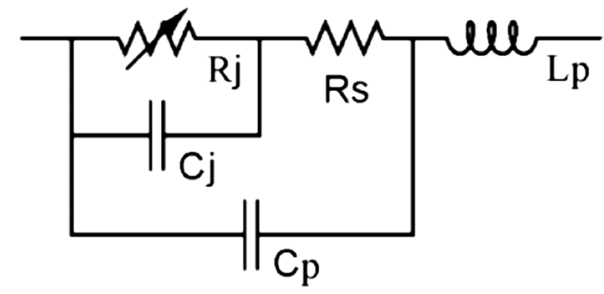

Fig. 1. Shockley diode model with package/pads parasitic components.

the diode zero-bias current responsivity. The parasitic efficiency can then be calculated by using [9]:

$$
\eta_{p}=\frac{1}{\left(1+\left(\omega_{0} \cdot C_{j 0}\right)^{2} \cdot R_{s} \cdot R_{j 0}\right)^{2}}
$$

where $\omega_{0}$ is the angular frequency of operation.

The matching network efficiency $\eta_{m}$ largely depends on the matching topology and circuit/component technology used to implement the matching network. However, (4) and (5) [14] can be used to estimate the relative increase or decrease of $\eta_{m}$ achieved with different candidate diodes, provided that the quality factor of the matching network $Q_{m}$ can be maintained at the same level. Such an assumption of constant $Q_{\mathrm{m}}$ is true if the source and load mismatch does not change substantially (i.e., the diode impedances under consideration are not dramatically different). Under these assumptions,

$$
\begin{aligned}
\eta_{m} & =\frac{1}{1+\frac{Q_{r}}{Q_{m}}} \\
Q_{r} & =\sqrt{\frac{R_{H I G H}}{R_{L O W}}-1}
\end{aligned}
$$

where $Q_{r}$ is the required quality factor, which depends on the mismatch between the highest and lowest impedances $\left(R_{H I G H}\right.$ and $R_{L O W}$, respectively) that are to be matched. In the case of diode-based matching for AMPH, this is typically the source impedance and the diode's $R_{j 0}$.

Using (1)through (5), one can understand the diode parameters that need to be optimized in order to maximize the power conversion efficiency.

As expected, equation (3) suggests that lowering the parasitic resistance and junction capacitance improves the parasitic efficiency. The importance of choosing devices with low parasitic capacitance is already well-known in the microwave community and will not be further discussed here.

The junction resistance $R_{j 0}$ has a more complex influence on AMPH performance. A higher $R_{j 0}$ increases the $\eta_{0} \cdot \eta_{d c T}$ efficiency, assuming that the load magnitude can be adjusted to match that of the junction resistance. However, $\eta_{p}$ exhibits the opposite trend, and decreases when $R_{j 0}$ is increased. Similarly, $\eta_{m}$ also degrades as $R_{j 0}$ increases since the losses in the matching network rise with an increased mismatch between source and diode junction resistance. The maximum PCE limitation that is reflected in the current-state-of-the-art AMPH designs [8], [9] resides in the fact that an optimum $R_{j 0}$ has been established, and for which $\eta_{0} \cdot \eta_{d c T} \cdot \eta_{p}$ reaches its maximum for Schottky diodes. 
Consequently, to improve power conversion efficiency at low incident power levels, the only remaining parameter to consider is the diode's zero-bias current responsivity $\Re_{I 0}$. However, as presented in the next section, Schottky diodes' $\Re_{I 0}$ is limited by thermionic emission over a potential barrier. This work explores and harnesses the use of another class of diodes, backward tunnel diodes, which are not governed by thermionic emission, for their use in AMPH applications.

\section{DiOdES FOR ENERGY HARVESTING}

Energy harvesting based on detection of low-level ac waveforms fundamentally relies on achieving a large second-order nonlinearity in order to maximize the RF-to-dc conversion. While large-signal rectification (i.e., switching) simply requires a strongly asymmetric I-V relationship to maximize the impedance difference between on- and off-states, harvesting energy from low-level sources that are insufficient to induce switching is more challenging. In this low-level signal regime, conversion efficiency is directly related to the square of the current responsivity $\Re_{I 0}$ as shown in (2), which equals half the curvature, $\gamma=\left(\partial^{2} l / \partial V^{2}\right) /(\partial I / \partial V)$, of the detection device at the zero-bias condition [8]. This metric can be directly assessed from the I-V characteristics of the device, and thus provides a convenient means for comparison among technologies. For thermionic devices such as Schottky and PN junctions for which the I-V relationship is of the form $J$ $=J_{O}(\exp (q V / n k T)-1)$, one can see that $\gamma=q / n k T$ $\approx 38.5 \mathrm{~V}^{-1}$ at room temperature, independent of technology or the details of device design. This imposes fundamental physical limits on the ultimately achievable performance of thermionic detectors for energy harvesting applications. To circumvent this limitation, devices based on alternative physical operation principles for which $\gamma$ is not limited to $q / k T$ are potentially attractive. One promising physical mechanism that can be leveraged to obtain a higher curvature is interband tunneling. In contrast to Schottky diodes, PN junctions, and transistor-based passive detectors (e.g. diode-connected FETs, Schottky diodes using HEMT gates) in which carriers in a single band interact with a voltage-controlled potential barrier as the basis for detection and rectification, interband tunnel diodes are governed by the combination of the density of states and occupancy probabilities on both sides of the tunnel junction as well as the tunneling probability. In this way, the density of states in both the conduction and valence bands, as well as the details of the tunnel barrier, can be used to engineer devices with increased second-order nonlinearity and thus offer the potential for improved detection and conversion efficiency. An analytical treatment of homojunction interband tunnel diodes can be found in [15]; within the simplified model used, an arbitrarily large curvature can be achieved by a proper selection of the Fermi levels within the device [15]. Fundamentally, these devices can offer larger curvatures than thermionic-emission based devices because the overlap between the occupied electron and hole densities of states is "filtered" by the bandgaps at the heterojunction, leading to a truncation of the Fermi-Dirac distribution for the carriers. Devices of this type have been demonstrated experimentally as millimeter-wave detectors [16], and curvatures as high as $70 \mathrm{~V}^{-1}$ have been reported experimentally [12], broadly consistent with the expectations with theory. It should be noted that in order to achieve these high curvatures, a "backward" tunnel diode structure is needed, in contrast to an Esaki diode [17], [18]. In the case of an Esaki diode, extremely high doping is used on both sides of the junction, leading to low junction resistance and the onset of negative differential resistance in the forward characteristic. For detector and harvester applications, however, the negative differential resistance is not desirable (the curvature changes sign at the onset of negative differential resistance, leading to a partial cancellation of the detected signal with an increasing input power) and the second-order curvature near zero bias is smaller than with the more modestly-doped backward diode structures [15].

However, homojunction tunnel diodes such as those described above also pose some challenges in energy harvesting applications. Of particular concern for RF and microwave applications, homojunction tunnel diodes have a large capacitance per unit area (as a direct consequence of the use of heavy doping to achieve the thin depletion region and band degeneracy required to enable tunneling). This limits the frequency range of application, due to increased parasitic losses, as well as the bandwidth achievable with reactive matching networks. It has also been reported that Ge-based tunnel junctions can pose reliability and manufacturability challenges [19].

As an alternative, heterostructure backward tunnel diodes are an attractive approach. These devices maintain the fundamental operational principles of homojunction tunnel diodes (interband tunneling) and thus the possibility for high curvature, while at the same time introducing significant additional degrees of freedom in the device design to allow optimization of the device performance for specific applications. For example, devices of this type have previously been demonstrated to provide high-sensitivity, low-noise microwave and millimeter-wave detection [11], [20], [21], [22] for applications such as passive millimeter-wave imaging, with an extremely low noise equivalent power (NEP) of $0.18 \mathrm{pW} / \mathrm{Hz}^{1 / 2}$ [11]. This record-low NEP is made possible by a combination of both extremely low device noise, in conjunction with higher curvature arising from the operational physics. To maximize performance for detection, the devices are designed to maximize the second order nonlinearity at zero bias, thereby allowing the detectors to be used without externally applied bias. This dramatically reduces $1 / \mathrm{f}$ noise (resulting in nearly thermal-noise-limited performance) [21], [23], and it has been shown that zero-bias second-order nonlinearity in excess of what is possible with Schottky diodes can be achieved [11]. This degree of control of the device characteristics is made possible by the substantial design flexibility in heterostructure devices. Low-level RF energy harvesters have similar requirements, i.e., strong nonlinearity near zero bias is needed for efficient RF-to-dc conversion, and thus these devices are also attractive for this application.

Fig. 2(a) shows a schematic cross-sectional diagram of the nominal heterostructure backward diode used in this work; the corresponding measured Current Density-Voltage characteristics are shown in Fig. 2(b). As can be seen, a rectifying characteristic is obtained, with an extremely asymmetric I-V characteristic and "knee" at very low voltage, indicative of a strong cur- 


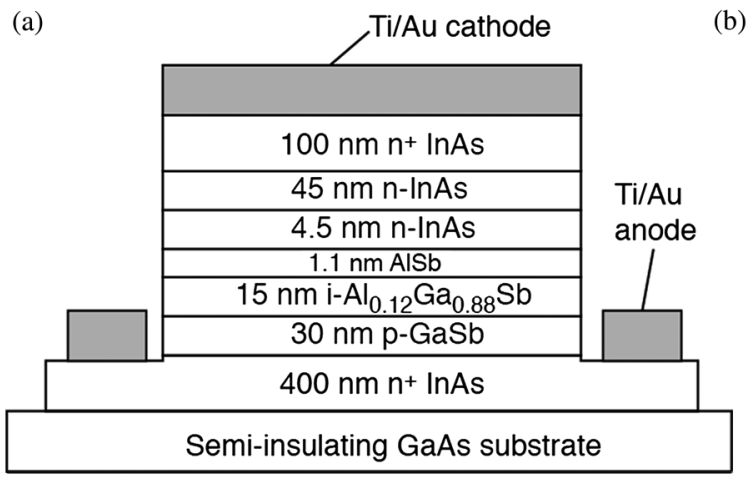

(c)

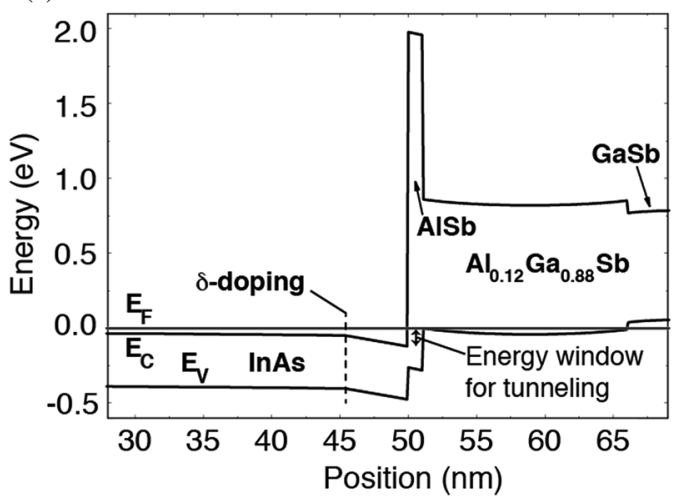

(b)

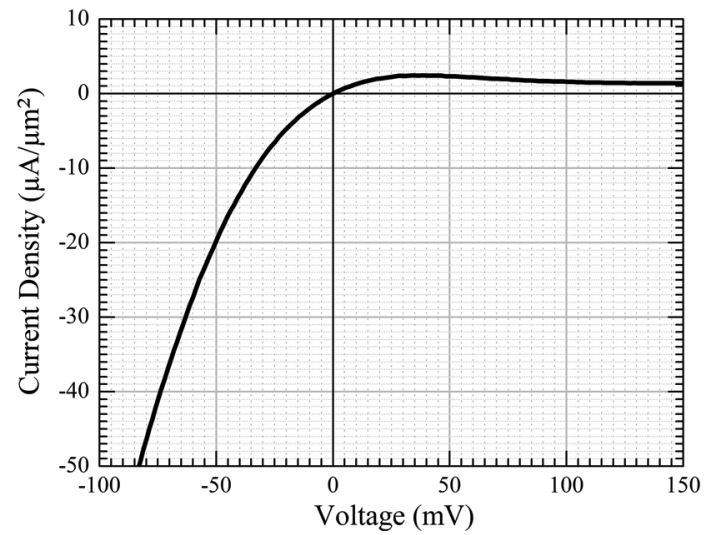

(d)

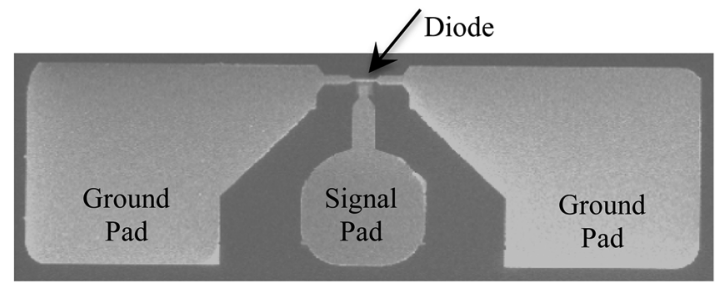

Fig. 2. (a) Schematic cross-section diagram for the heterostructure backward diode used in this work. The energy filtering enforced by the small tunneling window results in strongly nonlinear current-voltage characteristics and high sensitivity. (b) Current Density-Voltage characteristic for the heterostructure backward diodes used for the power harvesting prototypes presented in this work. (c) Calculated energy band diagram. (d) Scanning electron micrograph of fabricated backward tunnel diode with ground-signal-ground probe pads.

vature. In addition, the turn-on is in the 'reverse' direction (i.e., in the opposite sense from a conventional PN junction diode), as expected for a backward diode. As discussed previously, the efficiency of a power harvester is directly related to the junction resistance and curvature. Due to the interband tunneling mechanism in heterostructure backward diodes, $\gamma$ can be much larger than what is possible with conventional Schottky or other thermionic emission-based devices; optimizing a heterostructure backward tunnel diode for energy harvesting applications requires maximizing the curvature while keeping the junction resistance at an acceptably low level. The key parameters that enable control over the device performance are the anode and cathode doping concentrations (and doping profiles), the tunnel barrier thickness, and the energy band offset between the valence band in the anode and the conduction band in the cathode. As one example, Fig. 2(c) shows the computed energy band diagram for the backward diode structure shown in Fig. 2(a). The choice of an aluminum composition of $12 \%$ in the anode layer leads to a small overlap between the conduction and valence bands, and thus as shown Fig. 2(c), only carriers occupying a narrow range of energies can participate in tunneling through the AlSb tunnel barrier. Carriers with higher energies in either the anode or cathode are blocked by the bandgaps (i.e., are filtered) and cannot contribute to the current [15], [24]. The choice of the AlSb tunnel barrier thickness allows independent control of the junction resistance (since to first order tunneling prob- ability is exponential in barrier thickness) without altering the densities of states in the cathode and anode appreciably. The doping profiles in the anode and cathode are selected to enhance transmission through the structure, with a $\delta$-doping plane incorporated into the cathode in this structure to provide a nearly flat band profile.

The device heterostructure was grown by molecular beam epitaxy on a semi-insulating GaAs substrate. Device fabrication included evaporated Ti/Au anode and cathode contacts, mesa isolation using selective wet chemical etchants [25], and passivation with benzocyclobutene. The devices had an area of approximately $0.5 \mu \mathrm{m}^{2}$; electron beam lithography was used to accurately define the device active area. Optical lithography was used to define the pads, mesas, contacts, and passivation layers.

For the power harvesting experiments performed here, devices with the nominal heterostructure shown in Fig. 2(a) were used; this results in a measured zero-bias curvature $\gamma=45 \mathrm{~V}^{-1}$, and as will be shown, this improved curvature and low turn-on voltage result in high power harvester efficiency even at low incident RF levels. To further increase the harvester efficiency, increased curvature is desirable. Fundamentally, increases in curvature are correlated with improved energy filtering at the tunnel junction; one promising approach for narrowing the energy window over which tunneling can occur is by modifying the composition of the $\mathrm{AlGaSb}$ anode (i.e., increasing the $\mathrm{Al}$ composition results in a smaller tunneling window). Physics- 


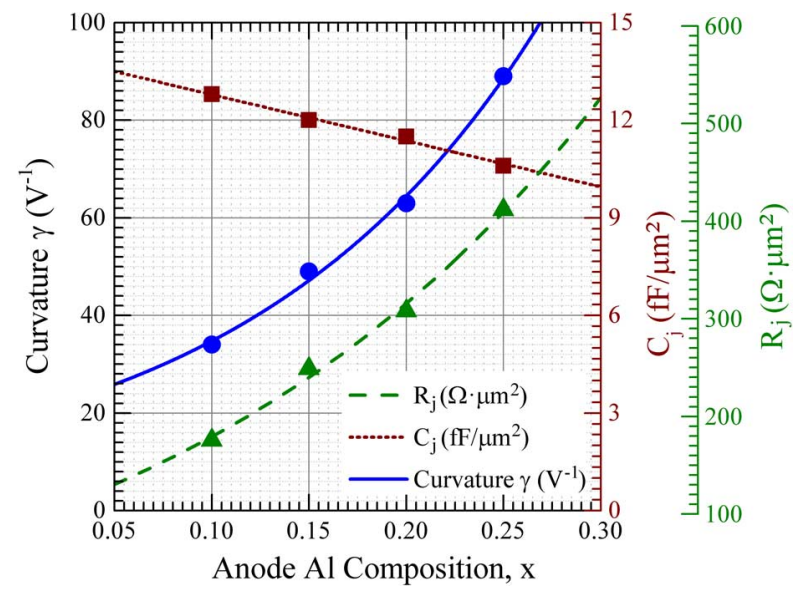

Fig. 3. Projected curvature $(\gamma)$ and junction resistance $\left(R_{j}\right)$ as a function of anode composition as obtained from physics-based simulations. Discrete points have been computed using the simulations; the curves are exponential fits to the simulated data. Increased curvature as well as junction resistance result from narrowing of the tunneling window with increasing Al composition in the anode.

based simulations of heterostructures with the same basic form as shown in Fig. 2(c), except with varying $\mathrm{Al}_{\mathrm{x}} \mathrm{Ga}_{1-\mathrm{x}} \mathrm{Sb}$ compositions, have been performed, and the junction resistance and curvature expected from these models is shown in Fig. 3. Details of the modeling framework have been reported previously [26]. As can be seen in Fig. 3, increasing the Al composition in the anode results in a significant increase in curvature, with curvatures approaching $90 \mathrm{~V}^{-1}$ possible for compositions of approximately $25 \%$. This increased curvature arises from the more narrowly-filtered carrier energy, but as expected this narrower energy range for tunneling is also accompanied by an increase in junction resistance. Our models project a harvester efficiency $\eta_{0} \cdot \eta_{d c T}$ of $63 \%$ for such a diode at $-40 \mathrm{dBm}$ input power, indicating that despite the promising results obtained to date and reported here, considerable additional improvements are possible. While further increases in curvature can be obtained, they come at the expense of a significant increase in junction resistance that may limit overall conversion efficiency. The junction resistance increase can be counterbalanced to some extent by increasing the diode's junction area, however at the cost of an increased junction capacitance. As a result, the optimum anode composition and junction area is also a function of the intended frequency of operation. Detailed study of these tradeoffs is the subject of ongoing work.

\section{BACKWARD TUNNEL DiOde CharacterizATION}

Backward tunnel diodes with $12 \%$ anode Al composition have been characterized using a $100 \mu \mathrm{m}$ pitch GSG (Ground-Signal-Ground) probe on a Cascade Summit probe station. A Keysight PNA network analyzer was used to measure the $S_{11}$ parameters and also as a calibrated microwave power source. The PNA network analyzer output power was calibrated at the probe $\mathrm{K}$-connector interface using a power meter, and the probe insertion loss was loaded into the network analyzer to compensate for power losses inherent from the probe. In this way, the power delivered to the diode could be precisely controlled. The diode sensitivity and I-V characteristics were measured using a 6-1/2 digit HP 34401A precision multimeter,

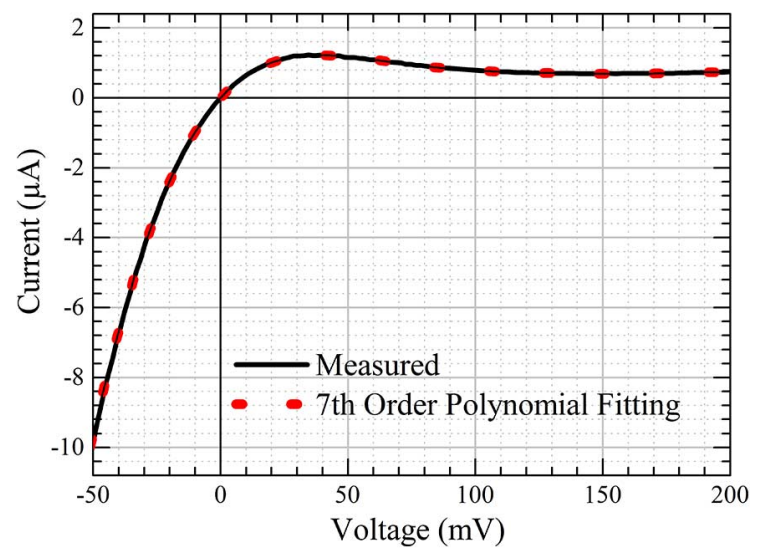

Fig. 4. Measured and modeled backward tunnel diode I-V curve.

connected to the network analyzer's internal bias-T. The dc current-voltage characteristic, as well as the diode's sensitivity and reflection coefficient $\Gamma$ from $500 \mathrm{MHz}$ to $40 \mathrm{GHz}$ were measured for an input power ranging from $-30 \mathrm{dBm}$ to -5 $\mathrm{dBm}$, in $5 \mathrm{~dB}$ steps.

The diode model parameters given in Fig. 1 were then extracted from the measurements. The nonlinear junction currentvoltage equation $I_{j}\left(V_{j}\right)$ was modeled using a 7 th order polynomial, given in (6); the coefficients were obtained through least-squares curve fitting to the measured I-V characteristics. A comparison between the modeling results and measurements is shown in Fig. 4. The dc non-linear model has been verified between $-100 \mathrm{mV}$ and $200 \mathrm{mV}$; this voltage range is sufficient to cover the voltages experienced by the device in ambient energy harvesting applications.

$$
\begin{aligned}
& I_{j}\left(V_{j}\right)=7.99 \cdot 10^{-5}\left(V_{j}\right)-1.73 \cdot 10^{-3}\left(V_{j}^{2}\right)+1.27 \cdot 10^{-2}\left(V_{j}^{3}\right) \\
& +6.76 \cdot 10^{-3}\left(V_{j}^{4}\right)-0.58\left(V_{j}^{5}\right)+2.92\left(V_{j}^{6}\right)-4.65\left(V_{j}^{7}\right)
\end{aligned}
$$

Using the $I_{j}\left(V_{j}\right)$ polynomial given in (6), the diode's junction resistance $R_{j}$, and current responsivity $\Re_{I}$ were calculated and are shown in Fig. 5 [27]. Having the non-linear junction behavior already defined, the measured sensitivity and reflection coefficient were used to extract the linear parasitic model parameters; these were found to be $C_{p}=13.7 \mathrm{fF}, R_{s}=25 \Omega$ and $L_{p}=52.6 \mathrm{pH}$. Although $C_{j}$ may in general be bias-dependent, for the model developed here $C_{j}$ was approximated by a linear (constant) capacitance of $4.5 \mathrm{fF}$. Modeling results and measurements are compared in Fig. 6. Although the measurements were limited to $40 \mathrm{GHz}$, the simulation results have been projected up to $100 \mathrm{GHz}$ to show the expected diode behavior.

The modeled diode resistance and responsivity near zero-bias are shown in Fig. 5, over the expected operating region for the AMPH application presented here. From this figure, a $\Re_{I 0}$ of $21.65 \mathrm{~A} / \mathrm{W}$ and $R_{j 0}$ of $12.5 \mathrm{k} \Omega$ are obtained for the particular backward tunnel diode used for this work.

Assuming that the parasitic efficiency is comparable for Schottky and tunnel diodes, the increase in the diode's $\Re_{I 0}$ from $19.34 \mathrm{~A} / \mathrm{W}$ in the ideal Schottky diode case to the measured tunnel diode responsivity of $21.65 \mathrm{~A} / \mathrm{W}$ indicates that the efficiency at square-law power levels is expected to increase by a factor of 1.25 according to (2). 


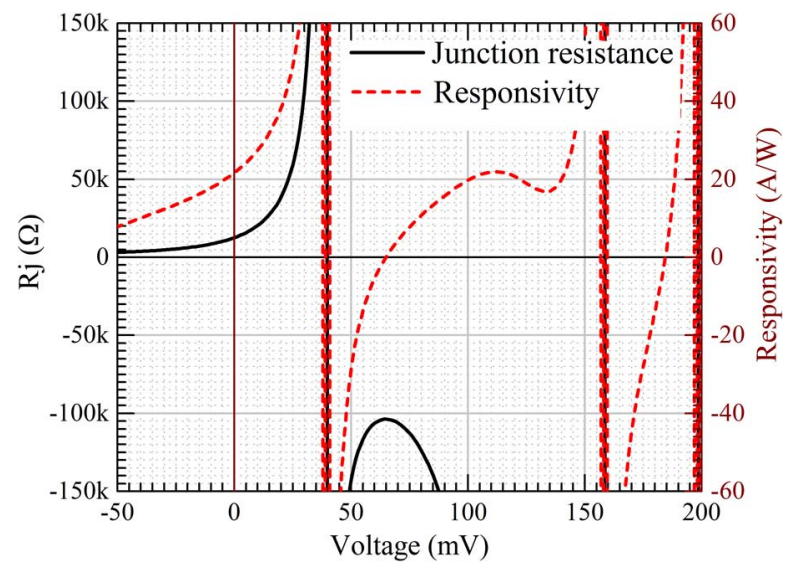

Fig. 5. Calculated backward diode $R_{j}$ and $\Re_{I}$, using the 7 th order polynomial fitted to the measured I-V relationship.
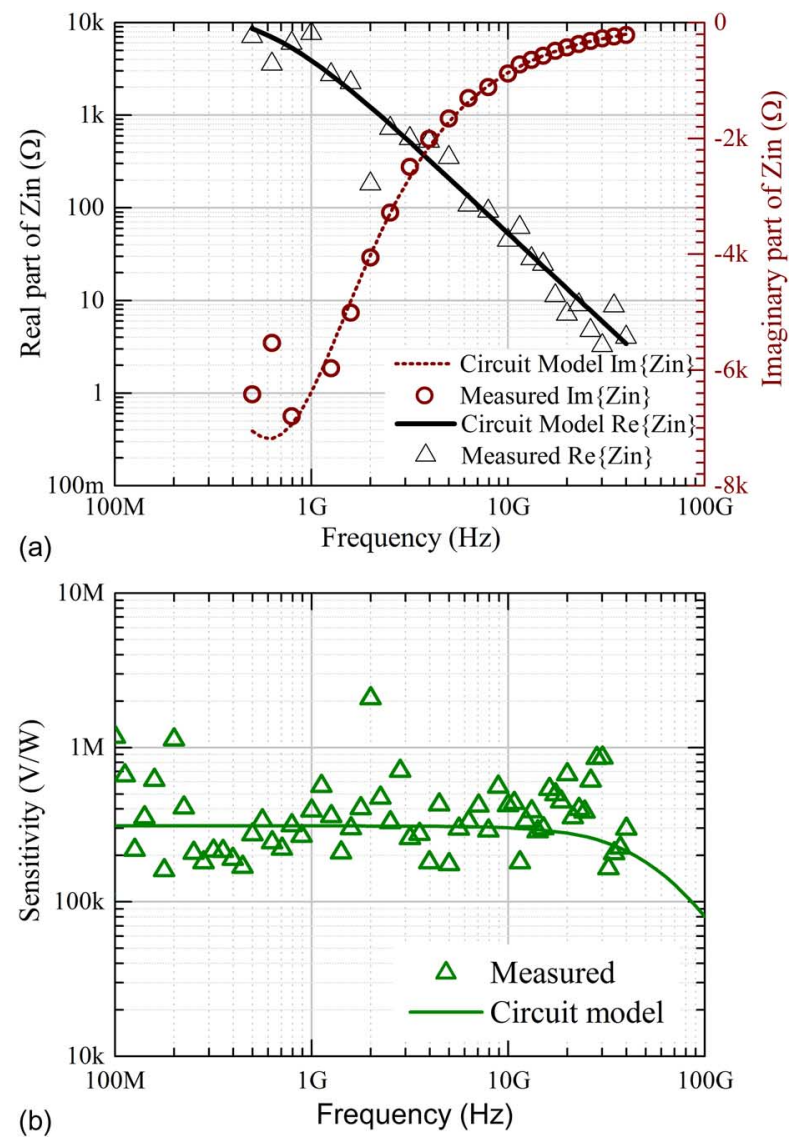

Fig. 6. Comparison between measured and modeled backward diode RF characteristics at $-30 \mathrm{dBm}$. (a) Input impedance, $Z_{\text {in }}$, real and imaginary components. (b) Voltage sensitivity; the simulation has been projected to $100 \mathrm{GHz}$.

\section{COMParison of BackWard TunNel Diode AND SCHOTTKY DiODE MiCROWAVE RECTIFIERS}

Neglecting the matching losses and considering that parasitic losses are negligible at $2.45 \mathrm{GHz}$ due to very low $C_{j}$, the studied backward diode has a calculated RF-to-dc power conversion efficiency equal to $14.7 \%$ at $-40 \mathrm{dBm}$ input power. Two commonly used Schottky diodes in AMPH applications were also evaluated and compared to the proposed backward diode, these
TABLE I

RECTIFIER EFFICIENCIES AT -40 DBM @ 2.4 GHz

\begin{tabular}{|l|c|c|c|c|c|c|}
\hline \multicolumn{1}{|c|}{ Diode } & $\Re_{I 0}$ & $R_{j 0}$ & $C_{j 0}$ & \multicolumn{4}{|c|}{ Efficiency (\%) } \\
\cline { 5 - 8 } & $(\mathrm{A} / \mathrm{W})$ & $\mathrm{k} \Omega$ & $\mathrm{fF}$ & $\boldsymbol{\eta}_{0} \boldsymbol{\eta}_{d c T}$ & $\boldsymbol{\eta}_{p}$ & PCE \\
\hline Backward & 21.65 & 12.5 & 4.5 & $\mathbf{1 4 . 7}$ & $\mathbf{9 9 . 7}$ & 14.6 \\
\hline Ideal Schottky & 19.34 & 12.5 & $\mathrm{NA}$ & $\mathbf{1 1 . 7}$ & $\mathrm{NA}$ & $\mathrm{NA}$ \\
\hline HSMS285B & 18.25 & 9.13 & 180 & $\mathbf{7 . 6}$ & 13.9 & 1.1 \\
\hline SMS7630 & 18.42 & 5.43 & 140 & $\mathbf{4 . 6}$ & $\mathbf{4 5 . 4}$ & 2.1 \\
\hline
\end{tabular}

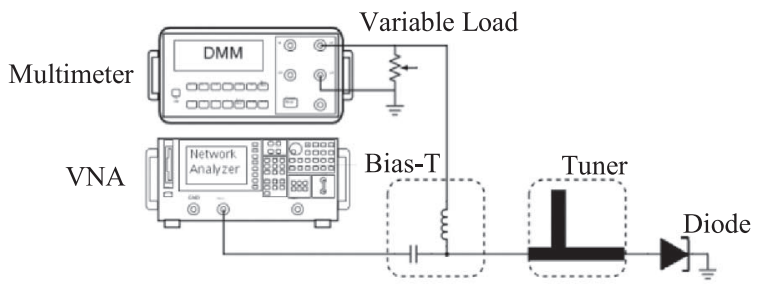

Fig. 7. Simplified schematic of the setup used for the microwave rectifier PCE measurements.

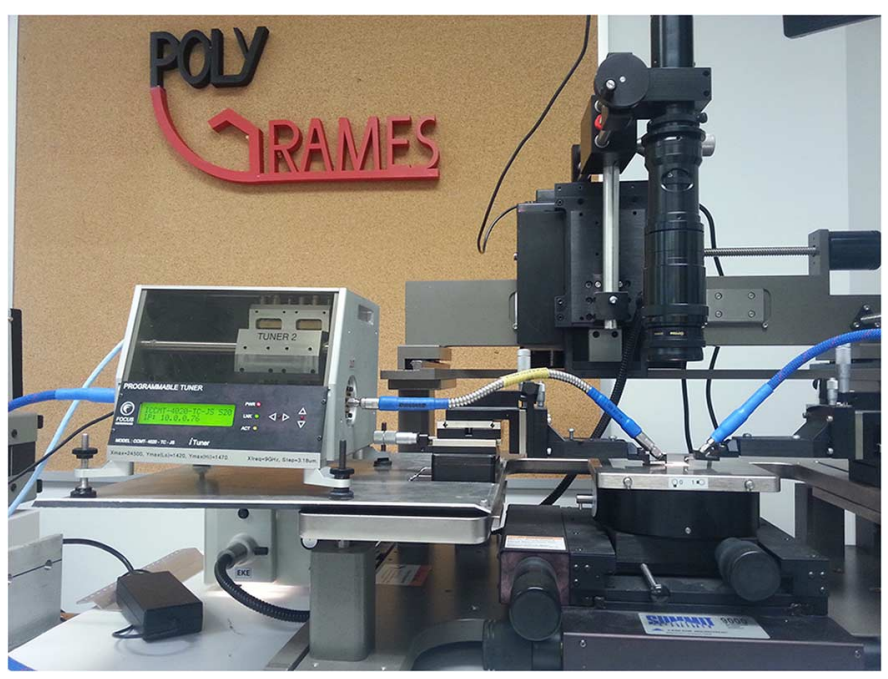

Fig. 8. Picture of part of the setup used to compare the PCE of the HSMS-2850 and backward tunnel diode. The picture shows the probe station and the Focus Microwaves Tuner, used as matching network.

are the Avago HSMS-2850 and the Skyworks SMS7630 diodes. Table I compares the expected power conversion efficiencies at $-40 \mathrm{dBm}$ input power, $2.4 \mathrm{GHz}$ center frequency, and resistive load matched to each diode's $R_{j 0}$. The efficiencies were calculated using the backward diode model parameters presented previously, and Schottky diodes parameters from the manufacturer datasheets. This comparison highlights two important advantages that arise from the use of the backward tunnel diode in AMPH applications. The first is the discussed increase in the $\eta_{0} \cdot \eta_{d c T}$ efficiency product due to the increased $\Re_{I 0}$. However, another advantage that should be noted is the very high parasitic efficiency due to the low junction capacitance that results from the backward tunnel diode structure used here.

From Table I, it can be seen that a large advantage is expected when using backward tunnel diode in AMPH applications; the expected PCE for the tunnel diode case is nearly 7 times the PCE of the SMS7630 diode at microwave input power levels typical of ambient harvesting applications. 


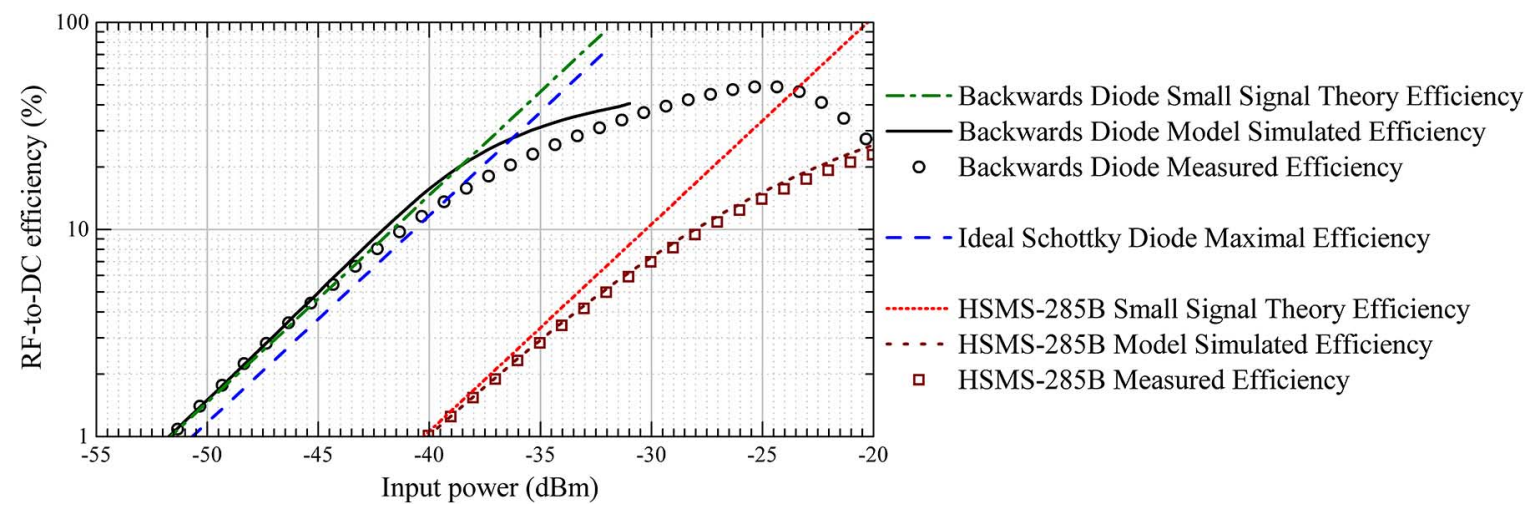

Fig. 9. Measured, simulated, and calculated efficiencies of the backward tunnel diode and HSMS-285B diode microwave rectifiers at 2.4 GHz. Matching network losses have been de-embedded.

In order to verify the increase in PCE predicted by the theory, both HSMS-285B and the backward diode rectification efficiencies were measured. The choice of the Schottky diode was based on the value of $R_{j 0}$, which should be near to that presented by the backward diode to ensure a valid comparison.

The microwave power rectification setup in our experiments consisted of a Keysight PNA Network Analyzer with an integrated bias-T, a Focus microwave tuner system used as matching network, and a 6-1/2 digit HP 34401A multimeter connected to a variable resistor, used as the dc load. The output power of the PNA was calibrated at the tuner input plane (source side) so that it could be used as the microwave power source. At the same time, the PNA was used to measure the reflection coefficient and ensure that a good matching was obtained at $2.4 \mathrm{GHz}$. A schematic diagram of the setup is shown in Fig. 7 and a picture in Fig. 8.

To evaluate the power incident on the diodes, the open-circuit detector voltage was measured, using the diodes as microwave power detectors. This information together with the diode's sensitivity measured during the characterization step was then used to extract the insertion losses of the matching circuit. In this way, the rectifier's RF-to-dc conversion efficiency $\eta_{0} \cdot \eta_{d c T}$ and parasitic efficiency $\eta_{P}$ were evaluated for both diode types, independently of any losses that originated from the matching circuit implementation.

For the efficiency measurement, the variable load was adjusted to its optimum value; $13 \mathrm{k} \Omega$ was used for the backward diode, and $9 \mathrm{k} \Omega$ for the HSMS-285B diode. The measured, calculated, and simulated results are given in Fig. 9 together with an indication of the maximum efficiency that an ideal Schottky diode with no parasitic losses and the same $R_{j 0}$ as the studied backward diode could attain. The simulation tool used was the Keysight ADS harmonic balance simulator.

The results given in Fig. 9 show the two predicted advantages of the evaluated backward diode for AMPH applications. The first is the improvement in power conversion efficiency due to the higher current responsivity. Even when compared to the ideal Schottky diode theoretical limit, the backward diode has a $25.3 \%$ higher efficiency at extremely low input power, showing a very good agreement with the previously calculated factor of 1.25 . The second advantage comes from the low junction capacitance resulting from the backward diode construc-

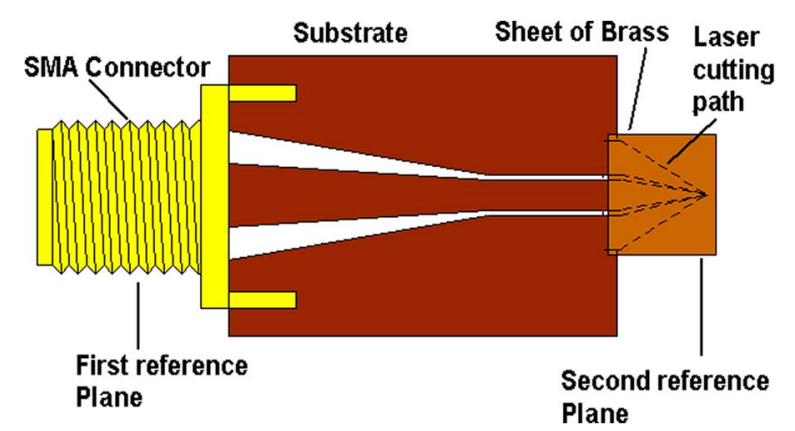

Fig. 10. An adapter characterization is done to extract the $S$ parameters of the probe, along with the substrate it is soldered on and the SMA connector.

tion. The resulting parasitic loss is extremely low at $2.4 \mathrm{GHz}$, leading to a 10.5 times higher efficiency when compared to the HSMS-285B diode at $-40 \mathrm{dBm}$ input power. This result too is in very good accordance with the previously calculated results given in Table I.

\section{BACKWARD TunNel Diode Rectifier Prototype}

In order to demonstrate a microwave power rectifier working at AMPH input power levels, a prototype with a narrow-band matching network has been fabricated to demonstrate operation in a more realistic context, including matching network losses. An in-house GSG probe was developed so multiple backward diodes could be tested using the same matching network board so the repeatability of the predicted result could be verified.

\section{A. GSG Probe Fabrication}

The GSG probes have been developed with the goal of being embeddable to any PCB circuit. For convenience, all test and qualification measurements have been done on the same $30 \mathrm{mil}$ Rogers RT/Duroid 6002 substrate. A 50 Ohm tapered coplanar line is used as a transition from the connector to the GSG probe, as seen in Fig. 10.

The GSG probe tips are realized on a thin 8 mil sheet of brass which is first welded to the PCB. The brass is then micromachined with a laser (indicated in Fig. 10 by the dotted lines) along the shape of the required tip. Alignment references are 


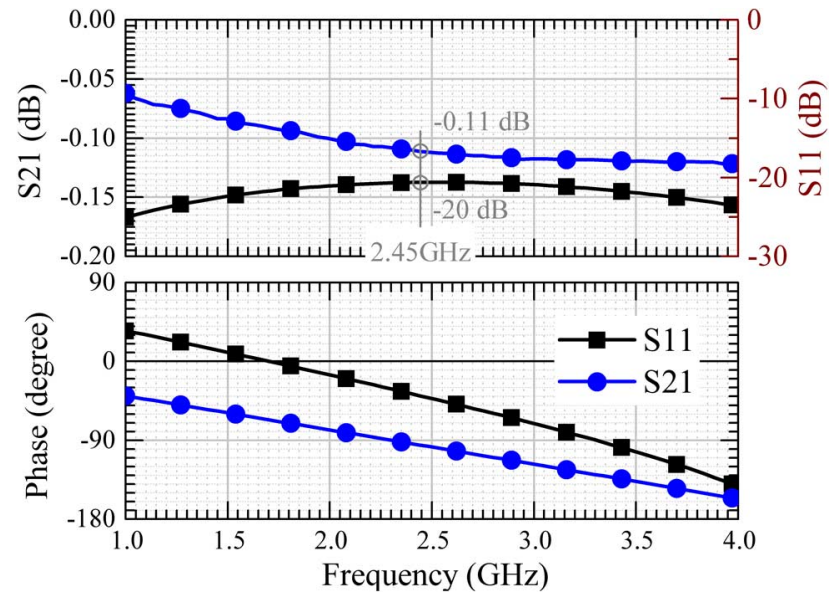

Fig. 11. Reflection and transmission parameter of the probe extracted through an adapter characterization procedure.

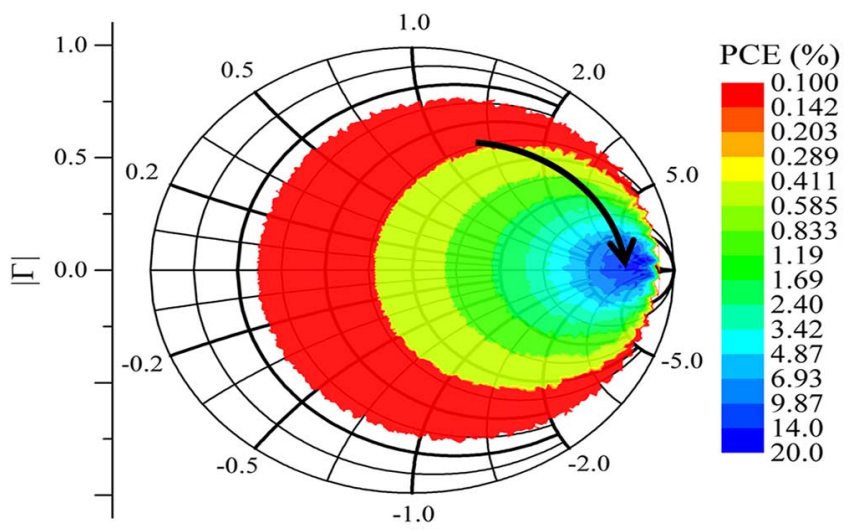

Fig. 12. Simulated PCE for different matching networks impedances. -30 $\mathrm{dBm}$ input power, $2.4 \mathrm{GHz}, 13 \mathrm{k} \Omega$ load. The arrow indicates an increasing PCE.

set from the sides of the transmission line to ensure the cutting contour fits the line and PCB gap.

The dimensions of the probe tip (starting pitch as well as the pitch of the tip) are determined on one side by our PCB fabrication process, which allows a minimum 5 mil gap between the ground plane and the 42 mil center conductor, and the on-wafer diode pads which have a $100 \mu \mathrm{m}$ pitch for the diodes reported here. A photograph of the micromachined probe tips can be seen in Fig. 13. To allow micro-positioning using standardized probe stations, a customized probe holder has been fabricated.

The scattering matrix has been extracted and validated using an adapter characterization procedure [28], [29], [30], which uses two calibration planes. The first reference plane is set with APC $3.5 \mathrm{~mm}$ calibration standards (before the connector) and the second reference plane is set at the end of the tips, with an on-wafer SOL calibration (using Picoprobe CS-5 standards). $\mathrm{S}_{21}$ and $\mathrm{S}_{11}$ are shown in Fig. 11.

\section{B. Rectifier Prototype Measurements}

The substrate used for the matching network construction is 30 mil Rogers RT/Duroid 6002. This design was based on the ideal matching network impedance given in Fig. 12, and includes a matching network with an adjustable length short cir-

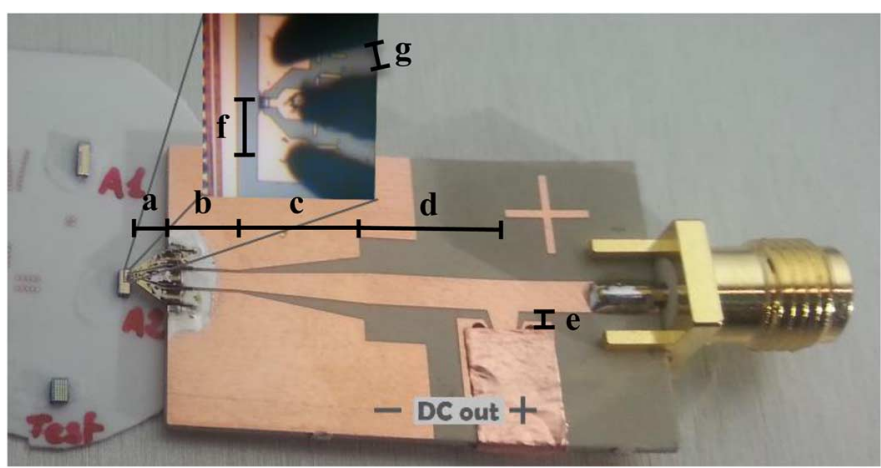

Fig. 13. Photograph of the rectifier circuit using a backward diode. The matching network was built and integrated with an in-house GSG probe used to interface with the diode pads (inset). $\mathrm{a}=83 \mathrm{mil}, \mathrm{b}=170 \mathrm{mil}, \mathrm{c}=300 \mathrm{mil}$, $\mathrm{d}=333 \mathrm{mil}, \mathrm{e}=25 \mathrm{mil}, \mathrm{f}=100 \mu \mathrm{m}, \mathrm{g}=2$ mil. Width of the line in $\mathrm{d}=75 \mathrm{mil}$, width of the line in $\mathrm{b}=42 \mathrm{mil}$ ( 6 mil separation between line and ground).

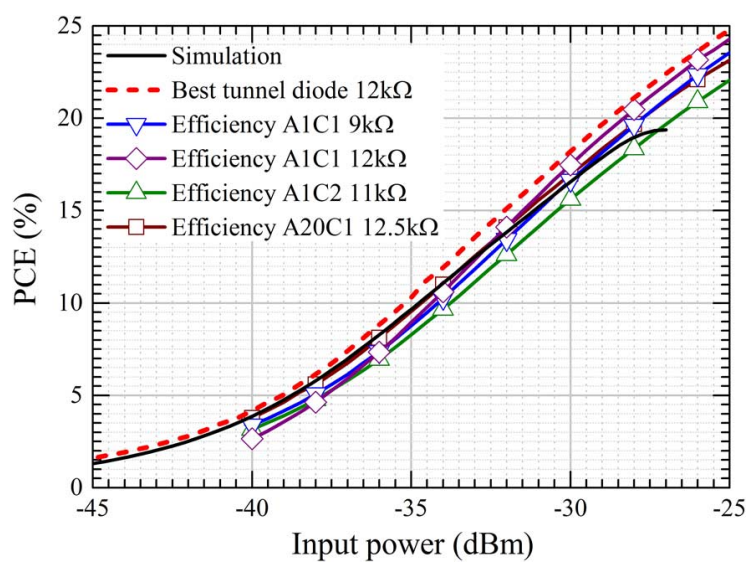

Fig. 14. Measured efficiencies for different diode samples at optimum load, the simulated result has been added for comparison.

cuit stub, which was used to fine-tune the narrow band matching network. A picture of the circuit is shown in Fig. 13.

Fig. 12 shows the PCE as a function of the matching network output impedance. The simulation was done using the backward diode model presented previously with an L-section matching network composed of a $50 \Omega$ series transmission line and $50 \Omega$ short-circuited stub which is used as the dc rectified current return path. The line and stub lengths were swept from $0.05 \mathrm{~mm}$ up to $\boldsymbol{\lambda} / 2$. The very high initial mismatch between the $50 \Omega$ source and the $12.5 \mathrm{k} \Omega$ diode $R_{j 0}$ introduces almost $3 \mathrm{~dB}$ matching network insertion loss, resulting in a reduced peak PCE of approximately $20 \%$ at $-30 \mathrm{dBm}$ input power, while the lossless case presented in Fig. 9 had a PCE near 37\% at the same frequency and input power. An important remark that can be made based on the results from Fig. 12 is that the impedance to be matched is nearly real, with very low imaginary part. This means that the proposed backward tunnel diodes may be matched to a large bandwidth using more complex matching network structures according to the Bode-Fano criterion [31].

Due to the $3 \mathrm{~dB}$ matching network insertion losses resulting from the high mismatch between the source and the diode junction resistance [32], an overall rectifier efficiency at $100 \mathrm{nW}$ of $3.8 \%$ has been measured. Although well below the $11.6 \%$ 


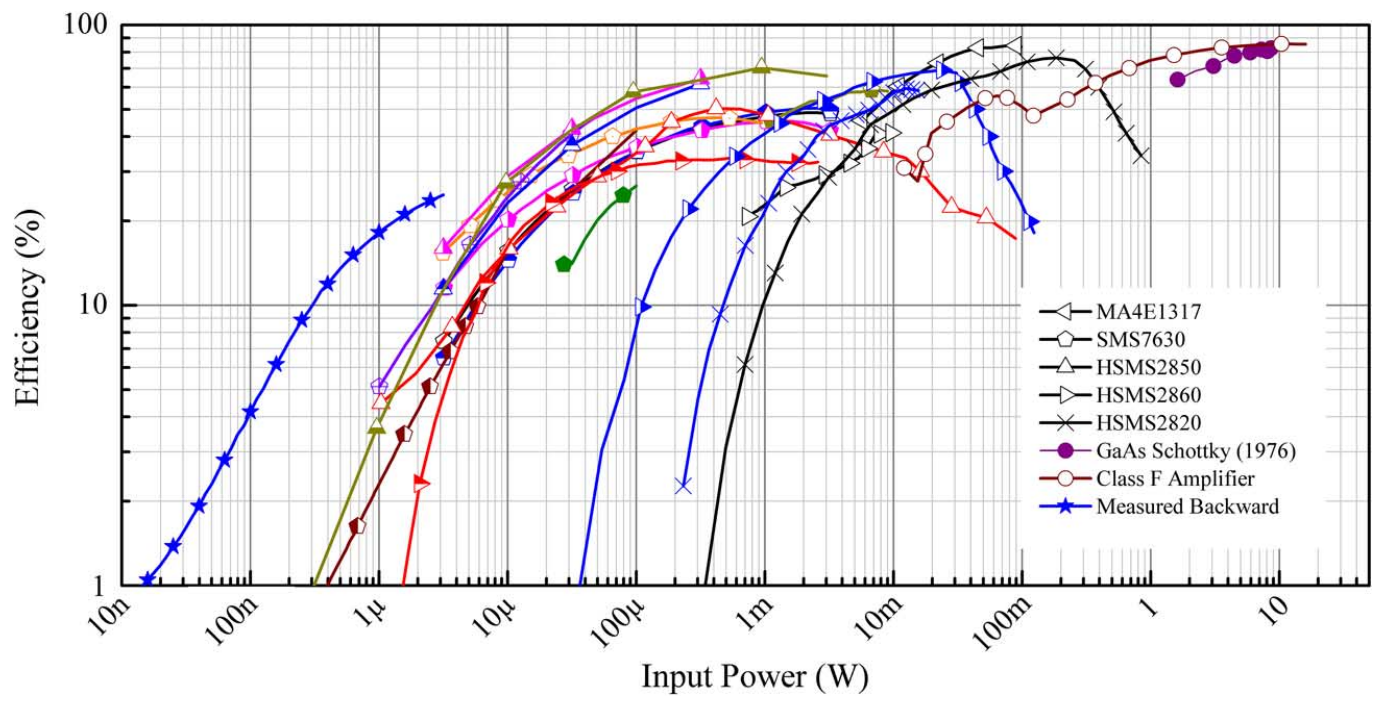

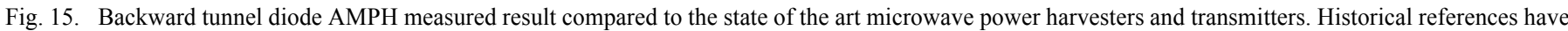
been added for reference. The symbol form has been maintained between rectifiers using the same diode.

TABLE II

REFERENCES From CURVES GIVEN IN FIG. 15

\begin{tabular}{|c|c|c|c|c|}
\hline Symbol & Diode & Frequency & Year & Source \\
\hline$\rightarrow \star$ & Backward Diode & $2.4 \mathrm{GHz}$ & 2015 & $\begin{array}{l}\text { This } \\
\text { Work }\end{array}$ \\
\hline$-1-$ & MA4E1317 & $2.45 \mathrm{GHz}$ & 2002 & {$[34]$} \\
\hline-0 & SMS7630 & $1.85 \mathrm{GHz}$ & 2010 & [35] \\
\hline 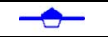 & SMS7630 & $2.45 \mathrm{GHz}$ & 2010 & [35] \\
\hline- & SMS7630 & $1.96 \mathrm{GHz}$ & 2012 & {$[36]$} \\
\hline$-1-$ & SMS7630 & $2.45 \mathrm{GHz}$ & 2010 & {$[37]$} \\
\hline$\rightarrow$ & SMS7630 & $2.45 \mathrm{GHz}$ & 2011 & {$[38]$} \\
\hline$\oplus$ & SMS7630 & $2.45 \mathrm{GHz}$ & 2014 & [39] \\
\hline$\triangle-$ & HSMS2850 & $1.8 \mathrm{GHz}$ & 2012 & [40] \\
\hline$\rightarrow$ & HSMS2850 & $2.15 \mathrm{GHz}$ & 2014 & [41] \\
\hline$-1-$ & HSMS2850 & $1.8 \mathrm{GHz}$ & 2014 & [41] \\
\hline$\Delta$ & HSMS2850 & $2.45 \mathrm{GHz}$ & 2013 & [42] \\
\hline$\rightarrow$ & HSMS2860 & $2.45 \mathrm{GHz}$ & 2010 & [43] \\
\hline$\rightarrow$ & HSMS2860 & $1.8 \mathrm{GHz}$ & 2012 & {$[40]$} \\
\hline$\rightarrow$ & HSMS 2820 & $1.8 \mathrm{GHz}$ & 2012 & {$[40]$} \\
\hline 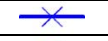 & HSMS2820 & $2.45 \mathrm{GHz}$ & 2010 & [44] \\
\hline- & GaAs Schottky & $2.388 \mathrm{GHz}$ & 1976 & [45] \\
\hline$-\infty$ & $\begin{array}{c}\text { Class F } \\
\text { Amplifier }\end{array}$ & $2.14 \mathrm{GHz}$ & 2012 & {$[46]$} \\
\hline
\end{tabular}

PCE expected from the measurements in Fig. 9, the device technology has proved to be promising based on this first demonstration. Fig. 14 shows the measured PCE for four different samples of the backward tunnel diode, showing the repeatability of the results across several tunnel diodes. The dc loads connected to the circuit were adjusted for each diode, in order to reach the maximum PCE. Measurements and Harmonic Balance (HB) simulation using the proposed backward tunnel diode model agree very well, indicating that the parameters extracted are in accordance with the actual diodes evaluated. The matching network was simulated using the Keysight Momentum electromagnetic fields simulator.

\section{CONCLUSION}

This paper has reported and demonstrated for the first time the possibility of overcoming the microwave power harvesting

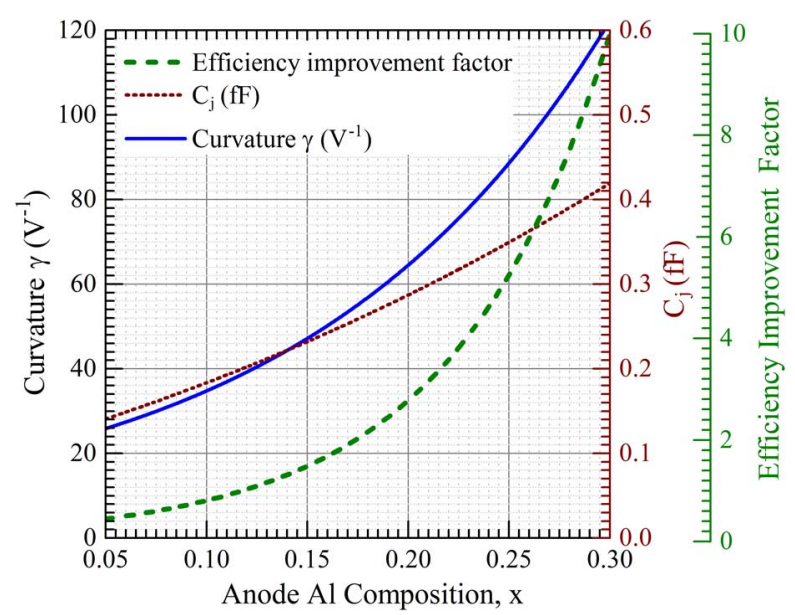

Fig. 16. Expected power conversion efficiency improvement for different backward tunnel diode anode Al compositions, the diode junction area was calculated to mantain a $12.5 \mathrm{k} \Omega$ junction resistance.

rectification efficiency limit of Schottky diode rectifiers, at low power levels typical of AMPH applications, through the use of backward tunnel diodes. A $25.3 \%$ increase in efficiency was observed when the backward diode rectifier model was compared to a theoretically ideal Schottky diode with the same $R_{j 0}$, while a 10.5 times higher efficiency was obtained when compared to a real HSMS-285B Schottky diode, due to lower diode parasitic losses and improved intrinsic current responsivity $\Re_{I 0}$.

The rectifier's measured efficiency for three different backward diodes agreed with the simulation results and showed a good repeatability between different backward diode devices. The best measured result from Fig. 14 is compared to other state-of-the-art published results in Fig. 15 and Table II; only diode based rectifiers operating between $1.5 \mathrm{GHz}$ and $3 \mathrm{GHz}$ were included in this comparison. The efficiency limitation of Schottky diode based AMPH is evident from this figure, even though different diodes, matching technologies and loads are 
used in the reports cited here, all of the Schottky-based rectifiers show an abrupt drop in the efficiency below approximately $10 \mu \mathrm{W}$ input power. The presented rectifier, based on a backward tunnel diode, on the other hand demonstrates good conversion efficiency for input powers in the $1 \mu \mathrm{W}$ range due to its low parasitic junction capacitance and increased current responsivity. The input power range for this figure has been extended to higher power levels to give the reader a good understanding of the power range where the backward tunnel diode can be used to increase microwave power transmission (MPT) and AMPH efficiency.

The backward diode rectifier efficiency roll off seen at higher powers likely originates from a larger voltage swing increasing the forward (thermionic) current, thereby limiting the efficiency. This could be addressed by using maximum power point tracking (MPPT) [33], rectification bridges using more diodes, or structural modifications to the diode design if input power is expected to reach higher values.

The results presented in this work were obtained using backward tunnel diodes optimized for low-level power detection and imaging applications. As shown in Fig. 3, the junction composition could be further optimized to increase the diode curvature $\gamma$, and consequently its current responsivity $\Re_{I 0}$, increasing the power conversion efficiency even more at lower input powers. The diode junction area, on the other hand, could be increased to optimize $R_{j 0}$, which could be used to minimize matching losses, although this would also increase the junction capacitance $C_{j}$. The authors believe this increase in $C_{j}$ would not be large enough to impact significantly performance in AMPH applications, since the frequencies of signals where higher power density appear are usually below $3 \mathrm{GHz}$ [5], [10]. The expected improvements in AMPH for a varying backward tunnel diode anode $\mathrm{Al}$ composition are shown in Fig. 16; this efficiency improvement was calculated using the simulation results given in Fig. 3 adjusting the junction area so that a $12.5 \mathrm{k} \Omega R_{j 0}$ was maintained. The intrinsic capacitance value remains negligible at $2.45 \mathrm{GHz}$ even with an increasing anode $\mathrm{Al}$ composition. As a result, the power conversion efficiency is improving in the same exponential fashion as the curvature $\gamma$. This low power analysis forecasts a significant improvement of at least one order of magnitude on the efficiency for narrower tunneling window. Determining and verifying the optimum characteristics of a backward tunnel diode for AMPH remains the subject of future work.

\section{ACKNOWLEDGMENT}

The authors would like to thank Rogers Corporation for providing the laminates used in this project.

The authors would also like to thank the support provided by the Poly-GRAMES Research Center technical support team, who are: Traian Antonescu, Steve Dubé, Maxime Thibault, and Jean-Sébastien Décarie.

\section{REFERENCES}

[1] D. Miorandi, S. Sicari, F. De Pellegrini, and I. Chlamtac, "Internet of things: Vision, applications and research challenges," Ad Hoc Netw., vol. 10, pp. 1497-1516, 2012.

[2] J. Gubbi, R. Buyya, S. Marusic, and M. Palaniswami, "Internet of Things (IoT): A vision, architectural elements, future directions," Future Generation Comput. Syst., vol. 29, pp. 1645-1660, 2013.
[3] Libelium, (2015, 15/06/2015), 50 Sensor Applications for a Smarter World [Online]. Available: http://www.libelium.com/ top_50_iot_sensor_applications_ranking/

[4] W. Wang, S. Hemour, and K. Wu, "Coupled resonance energy transfer over gigahertz frequency range using ceramic filled cavity for medical implanted sensors," IEEE Trans. Microw. Theory Techn., vol. 62, no. 4, pp. 956-964, Apr. 2014.

[5] K. Sangkil et al., "Ambient RF energy-harvesting technologies for selfsustainable standalone wireless sensor platforms," Proc. IEEE, vol. 102, no. 11, pp. 1649-1666, Nov. 2014.

[6] Z. Popovic, "Cut the cord: Low-power far-field wireless powering," IEEE Microw. Mag., vol. 14, no. 2, pp. 55-62, Mar.-Apr. 2013.

[7] D. Masotti, A. Costanzo, P. Francia, M. Filippi, and A. Romani, "A load-modulated rectifier for RF micropower harvesting with start-up strategies," IEEE Trans. Microw. Theory Techn., vol. 62, no. 4, pp. 994-1004, Apr. 2014.

[8] S. Hemour and $\mathrm{K}$. Wu, "Radio-frequency rectifier for electromagnetic energy harvesting: Development path and future outlook," Proc. IEEE, vol. 102, no. 11, pp. 1667-1691, Nov. 2014.

[9] S. Hemour et al., "Towards low-power high-efficiency RF and microwave energy harvesting," IEEE Trans. Microw. Theory Techn., vol. 62, no. 4, pp. 965-976, Apr. 2014.

[10] F. Giuppi, K. Niotaki, A. Collado, and A. Georgiadis, "Challenges in energy harvesting techniques for autonomous self-powered wireless sensors," in , 2013 Eur. Microw. Conf. (EuMC), 2013, pp. 854-857.

[11] Z. Zhang, R. Rajavel, P. Deelman, and P. Fay, "Sub-micron area heterojunction backward diode millimeter-wave detectors with 0.18 noise equivalent power," IEEE Microw. Compon. Lett., vol. 21, no. 5, pp. 267-269, May 2011.

[12] J. Karlovský, "The curvature coefficient of germanium tunnel and backward diodes," Solid-State Electron., vol. 10, pp. 1109-1111, 1967.

[13] C. H. P. Lorenz et al., "Overcoming the efficiency limitation of low microwave power harvesting with backward tunnel diodes," in 2015 IEEE MTT-S Int. Microw. Symp. Dig.,, May 2015, pp. 1-4.

[14] A. M. Niknejad, Electromagnetics for High-Speed Analog and Digital Communication Circuits. Cambridge, U.K.: Cambridge Univ. Press, 2007.

[15] S. M. Sze and K. K. Ng, Physics of Semiconductor Devices. Hoboken, NJ, USA: Wiley, 2006.

[16] C. Burrus, "Backward diodes for low-level millimeter-wave detection," IEEE Trans. Microw. Theory Techn., vol. 11, no. 5, pp. 357-362, Sep. 1963.

[17] L. Esaki, "New phenomenon in narrow germanium p-n junctions," Phys. Rev., vol. 109, p. 603, 1958.

[18] L. Esaki, "Discovery of the tunnel diode," IEEE Trans. Electron. Devices, vol. 23, no. 7, pp. 644-647, Jul. 1976.

[19] R. Meyers, P. Fay, J. Schulman, S. Thomas, III, D. Chow, and J. Zinck et al., "Bias and temperature dependence of Sb-based heterostructure millimeter-wave detectors with improved sensitivity," IEEE Electron. Device Lett., vol. 25, no. 1, pp. 4-6, Jan. 2004.

[20] J. Schulman et al., "Quantum tunneling Sb-heterostructure millimeterwave diodes," in IEDM Tech Dig., 2001, pp. 35.1. 1-35.1. 3.

[21] H. Moyer et al., "W-band Sb-diode detector MMICs for passive millimeter wave imaging," IEEE Microw. Compon. Lett., vol. 18, no. 10, pp. 686-688, Oct. 2008

[22] N. Su, R. Rajavel, P. Deelman, J. N. Schulman, and P. Fay, "Sb-heterostructure millimeter-wave detectors with reduced capacitance and noise equivalent power," IEEE Electron. Device Lett., vol. 29, no. 6, pp. 536-539, June 2008.

[23] J. J. Lynch et al., "Passive millimeter-wave imaging module with preamplified zero-bias detection," IEEE Trans. Microw. Theory Techn., vol. 56, no. 7, pp. 1592-1600, Jul. 2008.

[24] J. Schulman and D. Chow, "Sb-heterostructure interband backward diodes," IEEE Electron. Device Lett., vol. 21, no. 7, pp. 353-355, Jul. 2000.

[25] N. Su, Y. Tang, Z. Zhang, T. Kuech, and P. Fay, "Observation and control of electrochemical etching effects in the fabrication of InAs/ $\mathrm{AlSb} / \mathrm{GaSb}$ heterostructure devices," J. Vacuum Science \& Technol. B, vol. 26, pp. 1025-1029, 2008.

[26] M. Shams, I. Bin, Y. Xie, Y. Lu, and P. Fay, "An accurate interband tunneling model for InAs/GaSb heterostructure devices," Physica Status Solidi (c), vol. 10, pp. 740-743, 2013.

[27] H. C. Torrey, C. A. Whitmer, and S. A. Goudsmit, Crystal Rectifiers, 1st ed. New York, NY, USA: McGraw-Hill Book Co., 1948.

[28] Keysight, (2002, 29/06/2015), [VBA Sample Program] Adapter Characterization. User Manual No. 16000-95024 [Online]. Available: http://www.keysight.com/main/editorial.jsp $? \mathrm{cc}=\mathrm{VN} \& \mathrm{lc}=\mathrm{vie} \& \mathrm{ckey}=85082 \&$ nid $=-11143.0 .00 \& \mathrm{id}=85082$ 
[29] Agilent, (29/06/2015), Measuring Noninsertable Devices. Product Note No. 8510-13 [Online]. Available: http://cp.literature.agilent.com/litweb/pdf/5956-4373E.pdf

[30] J. Randa, W. Wiatr, and R. L. Billinger, "Comparison of adapter characterization methods," IEEE Trans. Microw. Theory Techn., vol. 47, no. 12, pp. 2613-2620, Dec. 1999.

[31] R. M. Fano, "Theoretical limitations on the broadband matching of arbitrary impedances," J. Franklin Inst., vol. 249, pp. 57-83, 1950.

[32] C. H. P. Lorenz, S. Hemour, and K. Wu, "Modeling and influence of matching network insertion losses on ambient microwave power harvester," presented at the Conf. Numerical Electromagnetic and Multiphysics Modeling and Optimization (NEMO), 2015 IEEE MTT-S Int., Ottawa, ON, Canada, 2015, unpublished.

[33] A. Dolgov, R. Zane, and Z. Popovic, "Power management system for online low power RF energy harvesting optimization," IEEE Trans. Circuits Syst. I, Reg. Papers, vol. 57, no. 7, pp. 1802-1811, Jul. 2010.

[34] Y.-H. Suh and K. Chang, "A high-efficiency dual-frequency rectenna for 2.45- and 5.8-GHz wireless power transmission," IEEE Trans. Microw. Theory Techn., vol. 50, no. 7, pp. 1784-1789, Jul. 2002.

[35] C. M. Ghiglino, "Ultra-Wideband (UWB) Rectenna Design for Electromagnetic Energy Harvesting," M.S. thesis, Dept. Teoria del Senyal i Comun., Escola Técnica Superior d'Enginyeria de Telecomun, de Barcelona, Catalunya, Spain, 2010.

[36] E. Falkenstein, M. Roberg, and Z. Popović, "Low-power wireless power delivery," IEEE Trans. Microw. Theory Techn., vol. 60, no. 7, pp. 2277-2286, Jul. 2012.

[37] G. Andia Vera, A. Georgiadis, A. Collado, and S. Via, "Design of a $2.45 \mathrm{GHz}$ rectenna for electromagnetic (EM) energy scavenging," in 2010 IEEE Radio and Wireless Symp. (RWS), 2010, pp. 61-64.

[38] K. Lui, A. Vilches, and C. Toumazou, "Ultra-efficient microwave harvesting system for battery-less micropower microcontroller platform," IET Microw., Antennas \& Propag., vol. 5, pp. 811-817, 2011.

[39] W. Haboubi et al., "An efficient dual-circularly polarized rectenna for RF energy harvesting in the $2.45 \mathrm{GHz}$ Ism band," Progress in Electromagn. Res., vol. 148, pp. 31-39, 2014.

[40] V. Marian, B. Allard, C. Vollaire, and J. Verdier, "Strategy for microwave energy harvesting from ambient field or a feeding source," IEEE Trans. Power Electron., vol. 27, no. 11, pp. 4481-4491, Nov. 2012.

[41] Z. Liu, Z. Zhong, and Y.-X. Guo, "High-efficiency triple-band ambient RF energy harvesting for wireless body sensor network," in $R F$ and Wireless Technologies for Biomedical and Healthcare Applications (IMWS-Bio), 2014 IEEE MTT-S Int. Microw. Workshop Series, 2014, pp. 1-3.

[42] B. R. Franciscatto, V. Freitas, J.-M. Duchamp, C. Defay, and T. P. Vuong, "High-efficiency rectifier circuit at $2.45 \mathrm{GHz}$ for low-inputpower RF energy harvesting," in 2013 Eur. Microw. Conf. (EuMC), 2013, pp. 507-510.

[43] S. Riviere, F. Alicalapa, A. Douyere, and J.-D. Lan Sun Luk, "A compact rectenna device at low power level," Progress in Electromagn. Res. C, vol. 16, pp. 137-146, 2010.

[44] H. Takhedmit et al., "A 2.45-GHz dual-diode RF-to-de rectifier for rectenna applications," in 2010 Eur. Microw. Conf. (EuMC), 2010, pp. $37-40$

[45] R. M. Dickinson, "Performance of a high-power, 2.388-GHz receiving array in wireless power transmission over $1.54 \mathrm{~km}$," in 1976 IEEE MTT-S Int. Microw. Symp., New York, NY, USA, 14-16, Jun. 1976, pp. $139-141$.

[46] M. Roberg, T. Reveyrand, I. Ramos, E. A. Falkenstein, and Z. Popovic, "High-efficiency harmonically terminated diode and transistor rectifiers," IEEE Trans. Microw. Theory Techn., vol. 60, no. 12, pp. 4043-4052, Dec. 2012.

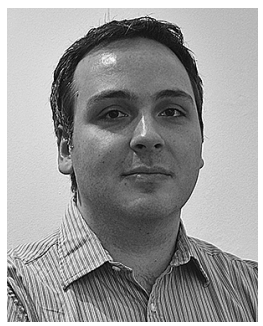

Carlos Henrique Petzl Lorenz (S'14) received the Dipl. Ing degree in electrical engineering from the Federal Technological University of Parana, Parana, Brazil, in 2007, and is concluding his Master studies at École Polytechnique de Montréal, Montreal, QC, Canada.

From 2007 to 2013, he was a Research and Development Engineer with Landis+Gyr Brazil, where he was responsible for the development of wireless communication interfaces. In 2013, he joined the Poly-Grames Research Center, École Polytechnique de Montréal. His research interests include microwave energy harvesting and power conversion and generation for low-power systems.

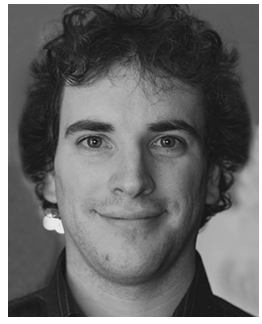

Simon Hemour (S'08-M'11) received the B.S. degree in electrical engineering from the University of Grenoble, Grenoble, France, in 2004 and the M.S. and Ph.D. degrees in optics, optoelectronics, and microwave engineering from the Grenoble Institute of Technology, Grenoble, France, in 2006 and 2010, respectively.

In 2003, he was with the European Organization for Nuclear Research (CERN), Geneva, Switzerland, as a part of the Instrumentation Department, where he was involved with the ATLAS experiment on the Large Hadron Collided (LHC). From 2006 to 2007, he was a Research Assistant with the Pidstryhach Institute of Applied Problems of Mechanics and Mathematics (IAPMM), National Academy of Science of Ukraine (NASU), Lviv, Ukraine. In 2007, he joined the IMEP-LAHC MINATEC Laboratory, Grenoble, France. From 2011 to 2015, he was with the Poly-Grames Research Center, Ecole Polytechnique de Montréal, Montréal, QC, Canada, leading the wireless power transmission and harvesting research group Since 2015, he has been with the University of Bordeaux, France, as an Assistant Professor of Electrical Engineering. His research interest include wireless power transfer and energy harvesting, ferrite-based RF circuits, nonlinear devices, innovative RF measurements, RF interferometry, low-power microwave, and millimeter-wave conversion circuits, and RF biomedical applications.

Dr. Hemour is a member of the IEEE MTT-26 "Wireless Energy Transfer and Conversion" Technical Committee.

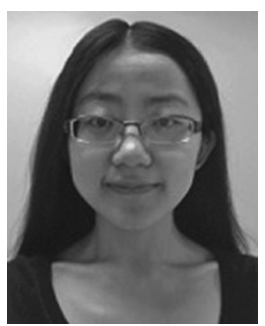

Wenjun Li received the B.S. degree in optoelectronics from Huazhong University of Science and Technology, Wuhan, China, in 2011. She is currently pursuing the Ph.D. degree in the Department of Electrical Engineering, University of Notre Dame, Notre Dame, IN, USA.

Her research interests include design, fabrication, and characterization of III-Nitride electronic devices, millimeter-wave detectors, III-V optoelectronics, and low power applications.

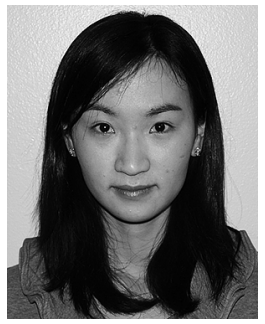

Yi Xi received the M.S. degree in electrical engineering from the University of Notre Dame, Notre Dame, IN, USA, in 2014.

In 2011, she joined Dr. Fay's group as a graduate student and her research was in the area of Sb-based millimeter-wave detectors. She is currently a Control Engineer with Cummins Inc., Columbus, IN, USA.

Jules Gauthier, photograph and biography not available at the time of publication.

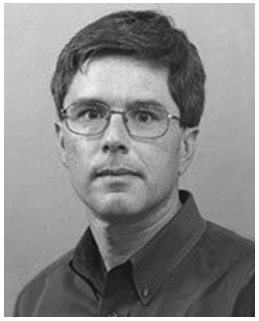

Patrick Fay (S'89-M'91-SM'02) received a Ph.D degree in electrical engineering from the University of Illinois at Urbana-Champaign, Urbana, IL, USA, in 1996.

He is a Professor in the Department of Electrical Engineering, University of Notre Dame, Notre Dame, IN, USA. His research interests include the design, fabrication, and characterization of microwave and millimeter-wave electronic devices and circuits, as well as high-speed optoelectronic devices and optoelectronic integrated circuits. His research also includes the development and use of micromachining techniques for the fabrication of microwave components and packaging. 


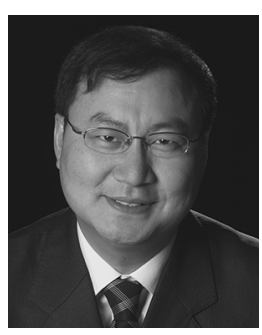

Ke Wu (M'87-SM'92-F'01) received B.Sc. degree (with distinction) in radio engineering from Nanjing Institute of Technology (now Southeast University), China, in 1982 and D.E.A. and Ph.D. degrees in optics, optoelectronics, and microwave engineering (with distinction) from the Institut National Polytechnique de Grenoble (INPG), Grenoble, France, and the University of Grenoble, Grenoble, France, in 1984 and 1987, respectively.

$\mathrm{He}$ is Professor of electrical engineering, and Tier-I Canada Research Chair in RF and millimeter-wave engineering at the Ecole Polytechnique (University of Montreal). He is the Director of the Poly-Grames Research Center. He was the founding Director of the Center for Radiofrequency Electronics Research of Quebec (Regroupement stratégique of FRQNT). He has also held guest, visiting and honorary professorship at many universities around the world. He has authored or co-authored over 1000 referred papers, and a number of books/book chapters and filed more than 30 patents. His current research interests involve substrate integrated circuits (SICs), antenna arrays, advanced CAD and modeling techniques, wireless power transmission and harvesting, and development of low-cost RF and millimeter-wave transceivers and sensors for wireless systems and biomedical applications. He is also interested in the modeling and design of microwave and terahertz photonic circuits and systems.

Dr. Wu is a member of Electromagnetics Academy, Sigma Xi Honorary Society, and URSI. He has held key positions in and has served on various panels and international committees including the chair of technical program committees, international steering committees and international conferences/symposia. In particular, he was the general chair of the 2012 IEEE MTT-S International Microwave Symposium. He has served on the editorial/review boards of many technical journals, transactions, proceedings and letters as well as scientific encyclopedia including editors and guest editors. He is currently the chair of the joint IEEE chapters of MTTS/APS/LEOS in Montreal. He is an elected IEEE MTT-S AdCom member for 2006-2015 and served as Chair of the IEEE MTT-S Transnational Committee, Member and Geographic Activities (MGA) Committee and Technical Coordinating Committee (TCC) among many other AdCom functions. He is the 2016 IEEE MTT-S President. He is the inaugural three-year representative of North America as Member of the European Microwave Association (EuMA) General Assembly. He was the recipient of many awards and prizes including the first IEEE MTT-S Outstanding Young Engineer Award, the 2004 Fessenden Medal of the IEEE Canada and the 2009 Thomas W. Eadie Medal of the Royal Society of Canada, the Queen Elizabeth II Diamond Jubilee Medal in 2013, the 2013 FCCP Education Foundation Award of Merit, the 2014 IEEE MTT-S Microwave Application Award, the 2014 Marie-Victorin Prize (Prix du Quebec-the highest distinction of Québec in the natural sciences and engineering), the 2015 Prix d'Excellence en Recherche et Innovation of Polytechnique Montréal, and the 2015 IEEE Montreal Section Medal of Achievement. He is a Fellow of the Canadian Academy of Engineering (CAE) and a Fellow of the Royal Society of Canada (The Canadian Academy of the Sciences and Humanities). He was an IEEE MTT-S Distinguished Microwave Lecturer from Jan. 2009 to Dec. 2011. 